\title{
Dos nuevas especies de Iridopsis Warren (Lepidoptera, Geometridae) del norte de Chile
}

\author{
Héctor A. Vargas ${ }^{1}$ \\ ${ }^{1}$ Departamento de Recursos Ambientales, Facultad de Ciencias Agronómicas, Universidad de Tarapacá, Casilla 6-D, Arica, Chile; Becario MECE \\ Educación Superior, Ministerio de Educación, Gobierno de Chile; Programa de Pós-Graduação em Entomologia, Universidade Federal do \\ Paraná, Curitiba-PR, Brasil. havargas@uta.cl
}

\begin{abstract}
Two new species of Iridopsis Warren (Lepidoptera, Geometridae) from northern Chile. Two new species of Iridopsis Warren, 1894, are described from northern Chile: I. hausmanni sp. nov. from the Azapa valley, Arica Province; and I. parrai sp. nov. from Pampa del Tamarugal, Iquique Province. The immature stages of I. parrai are associated with Prosopis tamarugo Phil. (Fabaceae). Photographs of the adults and figures of the male and female genitalia are included. This is the first mention of Iridopsis from Chile.
\end{abstract}

KEYWORDS. Ennominae; Boarmiini; Neotropical; Taxonomy.

RESUMEN. Dos nuevas especies de Iridopsis Warren (Lepidoptera, Geometridae) del norte de Chile. Se presentan descripciones de dos nuevas especies de Iridopsis Warren, 1894, del norte de Chile: I. hausmanni sp. nov., del valle de Azapa, Provincia de Arica; e I. parrai sp. nov., de la Pampa del Tamarugal, Provincia de Iquique. Los estados inmaduros de I. parrai se asocian con Prosopis tamarugo Phil. (Fabaceae). Esta es la primera mención de Iridopsis para Chile.

PALABRAS CLAVE. Ennominae; Boarmiini; Neotropical; Taxonomy.

Iridopsis se encuentra ampliamente distribuido a través del continente americano, y reúne actualmente a 93 especies descritas, 14 de ellas Neárticas y 79 Neotropicales (Pitkin 2002). Poco es conocido sobre los estados inmaduros de Iridopsis y su asociación con plantas hospederas (Passoa 1983). Sin embargo, Rindge (1966) ha indicado que los datos existentes para algunas especies Neárticas sugieren que estas son generalistas sobre plantas herbáceas, árboles deciduos y coníferos.

Pitkin (2002) ubicó a Iridopsis en la tribu Boarmiini (Ennominae), y sustentó la diagnosis y la monofilia de este género sobre la base de caracteres morfológicos de la genitalia del macho, principalmente la valva dividida y el amplio tegumen con "hombros", e indicó que la presencia de una mancha apical oscura sobre las alas anteriores es una ayuda para reconocer el género, aunque no está presente en todas las especies.

El objetivo de este trabajo es dar a conocer por primera vez la presencia de representantes de Iridopsis en Chile, mediante la descripción de dos nuevas especies colectadas en el extremo norte de este país.

\section{MATERIAL Y MÉTODO}

Los ejemplares examinados en este estudio fueron colectados entre los años 2001 y 2006, en las respectivas localidades: Valle de Azapa, Provincia de Arica, Chile, y Pampa del Tamarugal, Provincia de Iquique, Chile. Dos especímenes fueron obtenidos en laboratorio a partir de larvas folívoras colectadas sobre Prosopis tamarugo Phil. (Fabaceae) en la Pampa del Tamarugal. En las descripciones ha sido seguida la terminología de McGuffin (1987), y se ha empleado el término "hombros" mencionado por Pitkin (2002) para referirse a pequeñas proyecciones posteriores ubicadas sobre el tegumen en la genitalia del macho. El material tipo ha sido depositado en el Museo Nacional de Historia Natural de Santiago (MNNC), Santiago, Chile, en el Museo de Zoología de la Universidad de Concepción (UCCC), Concepción, Chile, y en la Colección Entomológica de la Universidad de Tarapacá (IDEA), Arica, Chile.

\section{RESULTADOS}

Iridopsis hausmanni sp. nov. (Figs. 1, 3, 4, 5, 6, 7, 8)

Holotipo macho, CHILE, Arica: Azapa, Arica, Chile, agosto 2006, H.A. Vargas (MNNC). Paratipos: CHILE, Arica, 1 macho, 1 hembra, Azapa, Arica, Chile, febrero 2006, H.A. Vargas (MNNC); 2 machos 1 hembra Azapa, Arica, Chile, febrero 2006, H.A. Vargas (UCCC); 1 macho Azapa, Arica, Chile, mayo 2003, H.A. Vargas; 1 macho Azapa, Arica, Chile, julio 2006, H.A. Vargas; 1 macho Azapa, Arica, Chile, junio 2004, H.A. Vargas; 1 macho Azapa, Arica, Chile, junio 2006, H.A. Vargas; 2 machos mismos datos holotipo; 1 hembra Azapa, Arica, Chile, febrero 2006, H.A. Vargas; 1 hembra Azapa, Arica, Chile, enero 2001, H.A. Vargas (IDEA).

Diagnosis. Fácilmente reconocible por la presencia de cuatro cornuti elongados de gran tamaño sobre la vesica en la genitalia del macho, los cuales tienen forma de $\mathrm{V}$ en sección transversal; y por la presencia de una mancha apical blanca sobre la superficie ventral del ala anterior, rodeada por escamas grisáceas a lo largo del margen interno. 

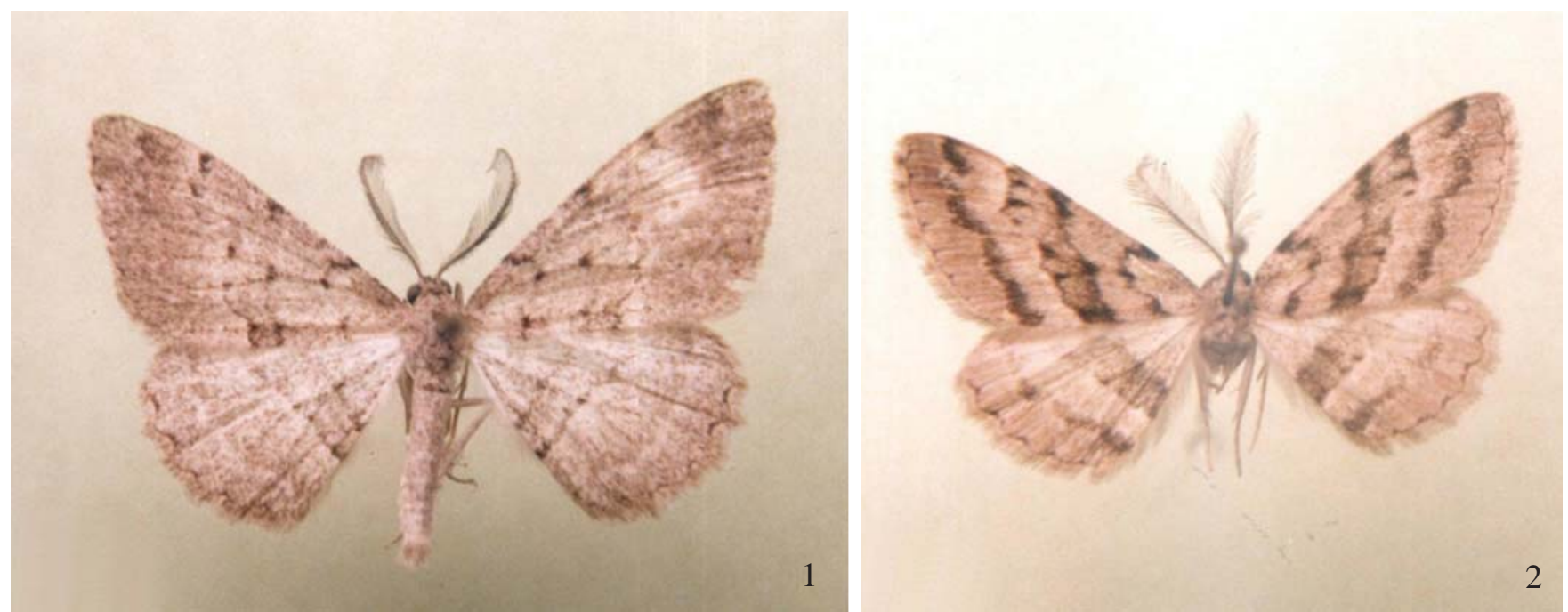

Fig 1-2. Especies de Iridopsis del norte de Chile. 1) I. hausmanni, macho en vista dorsal; 2) I. parrai, macho en vista dorsal.

Macho. (Fig. 1) Cabeza con ojos compuestos de color gris oscuro; frente parda grisácea, con una franja parda blanquecina sobre el margen ventral; vértex pardo blanquecino con algunas escamas pardas grisáceas dispersas; occipucio pardo blanquecino; antena bipectinada, escapo, pedicelo y dorso del flagelo cubiertos de escamas pardas blanquecinas; palpo labial pequeño, pardo blanquecino en la base, pardo grisáceo distalmente. Tórax cubierto de escamas pardas blanquecinas, algunas escamas piliformes ubicadas sobre las tégulas y lateralmente sobre el resto del tórax. Ala anterior: superficie dorsal parda blanquecina con escamas pardas grisáceas dispersas; línea basal, antemedial y medial sinuosas, de color pardo grisáceo, débilmente diferenciadas; línea subterminal blanca grisácea y sinuosa; línea adterminal parda grisácea débilmente diferenciada; superficie ventral parda blanquecina; presenta una mancha distal blanca rodeada por escamas grisáceas a lo largo del margen interno; mancha discal parda grisácea; banda subterminal parda grisácea. Ala posterior similar al ala anterior, pero carece de línea basal. Pata protorácica presenta epífisis tibial; superficie anterior de la coxa, fémur y tibia parda grisácea, el resto pardo blanquecino con algunas escamas pardas grisáceas dispersas. Pata mesotorácica con un par de espinas sobre la tibia; coloración similar a la de la pata protorácica. Pata metatorácica con dos pares de espinas tibiales; coloración parda blanquecina con algunas escamas pardas grisáceas dispersas, pincel de escamas piliformes sobre las tibias ausente. Abdomen pardo blanquecino, con algunas escamas pardas grisáceas dispersas; hilera de escamas piliformes sobre esternito III ausente.

Genitalia del macho (Fig. 3,4,5). Uncus corto, ápice simple; gnathos ausente; tegumen amplio, margen anterior profundamente hendido, margen posterior con "hombros"; saccus estrecho, membranoso ventralmente; juxta en forma de $\mathrm{Y}$, base ligeramente ensanchada y proyectada anteriormente, márgenes laterales sinuosos, margen distal hendido; valva profundamente dividida, lóbulo costal amplio con el margen distal ampliamente hendido, sacculus de ápice estrecho, presenta una débil proyección subapical; aedeagus subcilíndrico, elongado y largo, vesica con cuatro cornuti elongados de gran tamaño, con forma de $\mathrm{V}$ en sección transversal, la longitud del mayor sobrepasa a la mitad de la longitud del aedeagus.

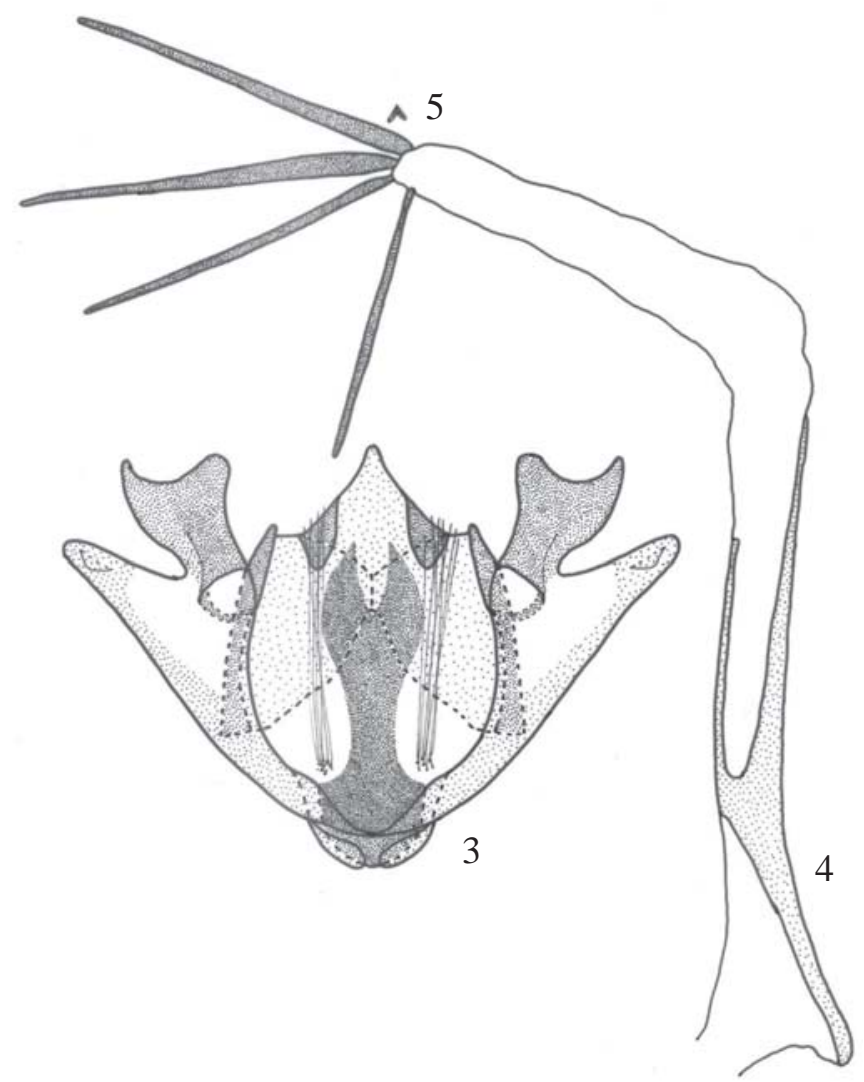

Figs. 3-5. Genitalia del macho de I. hausmanni. 3) Vista ventral, aedeagus removido; 4) aedeagus en vista lateral; 5) corte transversal del cornutus. 


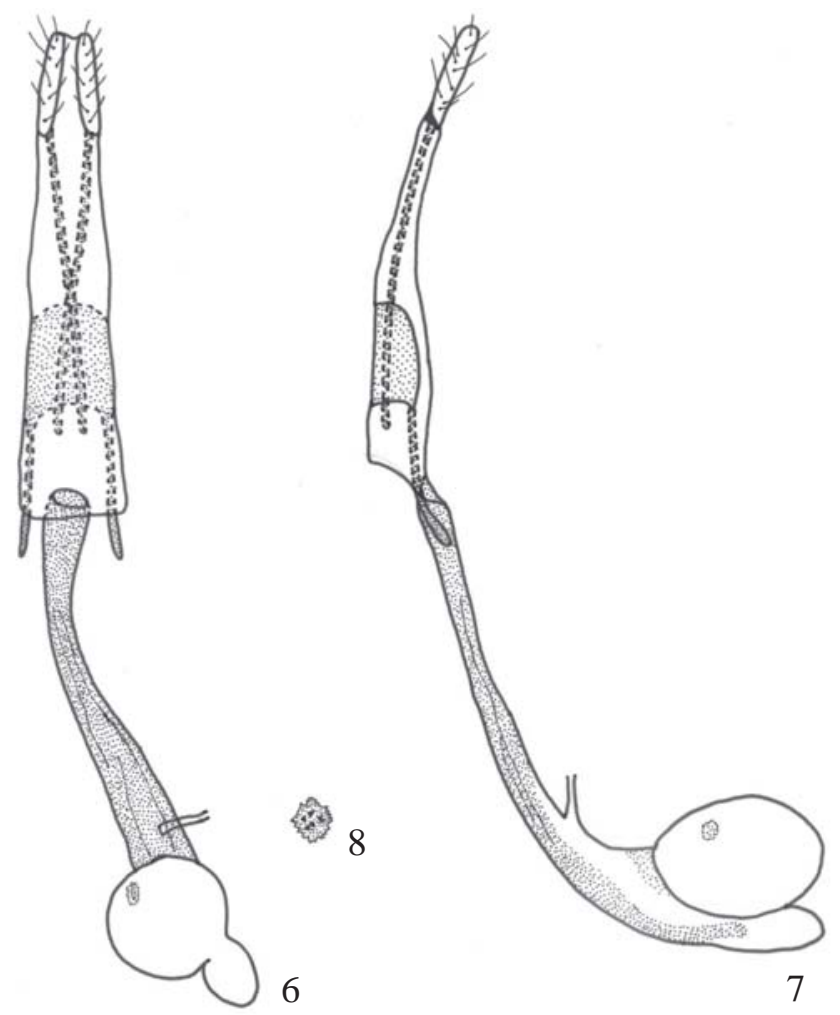

Figs. 6-8. Genitalia de la hembra de I. hausmanni. 6) Vista ventral; 7) vista lateral; 8) signum.

Hembra. Similar al macho, pero con antenas filiformes.

Genitalia de la hembra (Fig. 6,7,8). Ductus bursae subcilíndrico, elongado, débilmente sinuoso, esclerosado, con un área membranosa apical, y otra ventral rodeando la inserción del ductus seminalis; corpus bursae membranoso, inserto ventralmente sobre el cuarto distal del ductus bursae, no sobrepasa el ápice del ductus bursae; signum estelado con abundantes procesos espiniformes pequeños y esclerosados.

Distribución. Conocida sólo de la localidad tipo: Azapa (18 $\left.34^{\circ} \mathrm{S} ; 70^{\circ} 00^{\prime} \mathrm{W}\right)$, Arica, norte de Chile.

Planta hospedera. Desconocida.

Etimología. El nombre de esta especie está dedicado al Dr. Axel Hausmann, Zoologische Staatssammlung Muenchen, Alemania, por su valioso aporte al estudio de Geometridae.

Iridopsis parrai sp. nov.

(Figs. 2, 9, 10, 11, 12)

Holotipo macho, CHILE, Iquique: Refresco, Pampa Tamarugal, Iquique, Chile, Noviembre 2001, H.A. Vargas (MNNC); Paratipos: CHILE, 1 macho, 2 hembras mismos datos holotipo (MNNC); 2 machos, 2 hembras mismos datos holotipo (UCCC); 2 machos, 2 hembras mismos datos holotipo, 2 machos mismos datos holotipo, y con una etiqueta extra indicando "ex larva en Prosopis tamarugo" (IDEA).

Diagnosis. Notablemente similar a I. hausmanni, pero presenta bandas bien diferenciadas sobre la superficie dorsal de las alas, y carece de mancha distal blanca sobre la superficie ventral del ala anterior. Además, el aedeagus presenta cornuti subcilíndricos de pequeño tamaño en la vesica de la genitalia del macho, y la genitalia de la hembra carece de signum sobre el corpus bursae.

Macho (Fig. 2). Cabeza con ojos compuestos de color gris oscuro; frente, vértex, occipucio y palpos cubiertos por escamas de color pardo rojizo; antena bipectinada, escapo, pedicelo y dorso del flagelo cubiertos de escamas pardas rojizas. Tórax pardo rojizo, con algunas escamas piliformes sobre las tégulas y lateralmente sobre el resto del tórax. Ala anterior: superficie dorsal parda rojiza con escamas pardas grisáceas dispersas; línea basal, antemedial, subterminal y adterminal bien diferenciadas, sinuosas y de color pardo grisáceo; abundantes escamas pardas grisáceas presentes entre la línea antemedial y medial; superficie ventral parda blanquecina; mancha discal parda grisácea débilmente diferenciada. Ala posterior: superficie dorsal similar a la del ala anterior, pero carece de línea basal, las restantes líneas están débilmente diferenciadas cerca de la costa. Superficie ventral similar a la del ala anterior. Pata protorácica presenta epífisis tibial; superficie anterior de la coxa y fémur parda rojiza, el resto pardo blanquecino. Pata mesotorácica parda blanquecina; presenta un par de espinas tibiales: Pata metatorácica parda blanquecina; presenta dos pares de espinas tibiales; pincel de escamas piliformes sobre las tibias ausente. Abdomen pardo rojizo con escamas pardas grisáceas dispersas; hilera de escamas piliformes sobre esternito III ausente.

Genitalia del macho (Fig. 9-10). Uncus corto, ápice simple; gnathos ausente; tegumen amplio, margen anterior profundamente hendido, margen posterior con "hombros"; saccus estrecho, membranoso ventralmente; juxta en forma de Y, base ligeramente ensanchada y proyectada anteriormente, márgenes laterales sinuosos, margen distal hendido; valva profundamente dividida, lóbulo costal amplio con el margen distal ampliamente hendido, sacculus de ápice estrecho;

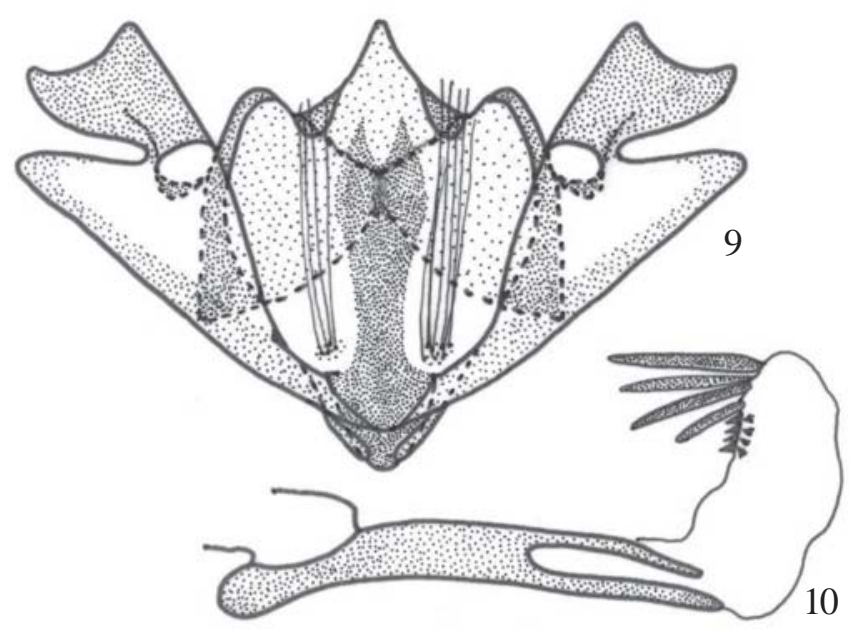

Figs. 9-10. Genitalia del macho de I. parrai. 9) Vista ventral, aedeagus removido; 10) aedeagus en vista lateral. 

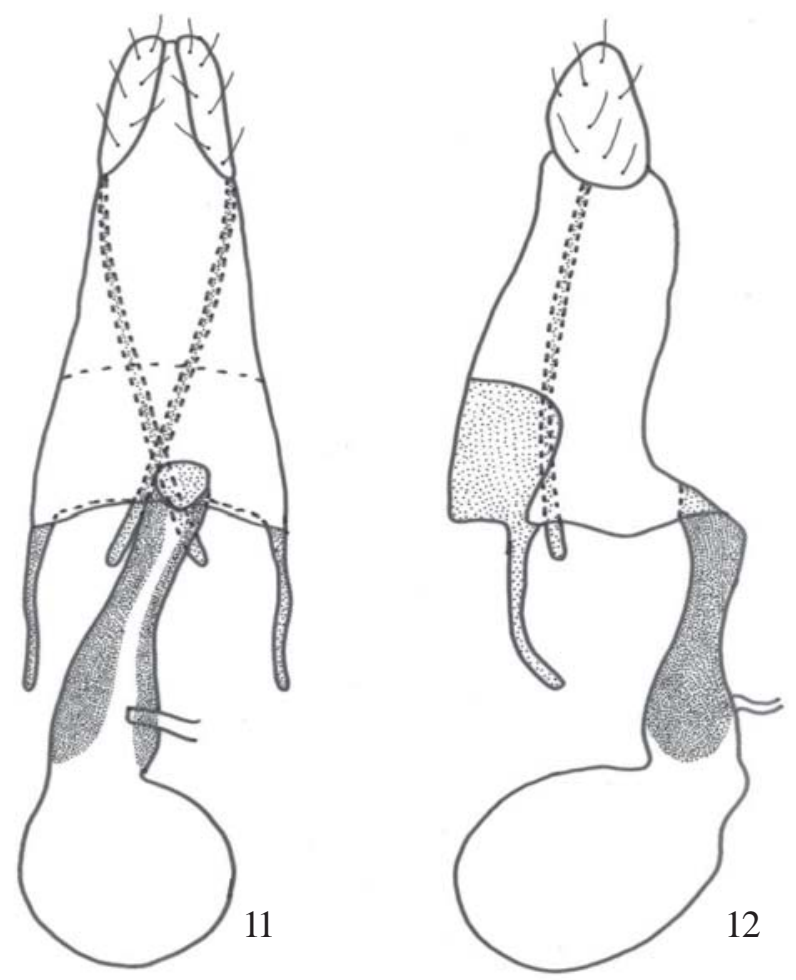

Figs. 11-12. Genitalia de la hembra de I. parrai. 11) Vista ventral; 12) vista lateral.

aedeagus subcilíndrico y elongado, vesica con cuatro cornuti subcilíndricos elongados de tamaño menor a la mitad de la longitud del aedeagus, y abundantes cornuti escuamiformes pequeños.

Hembra. Similar al macho, pero con antenas filiformes.

Genitalia de la hembra (Fig. 11-12). Ductus bursae esclerosado, subcilíndrico, elongado, ligeramente ensanchado cerca del ápice, presenta una estrecha franja membranosa longitudinal a lo largo de la línea media ventral; corpus bursae membranoso, subesférico, inserto sobre el ápice del ductus bursae; signum ausente; ductus seminalis inserto ventralmente cerca del ápice del ductus bursae.

Distribución. Conocida sólo de la localidad tipo: Pampa del Tamarugal $\left(20^{\circ} 21^{\prime} \mathrm{S} ; 69^{\circ} 39^{\prime} \mathrm{W}\right)$, Provincia de Iquique, norte de Chile.

Planta hospedera. Las larvas de esta I. parrai se alimentan de folíolos de "tamarugo" Prosopis tamarugo Phil. (Fabaceae).
Etimología. El nombre de esta especie está dedicado al Dr. Luis E. Parra, Universidad de Concepción, Chile, por su valioso aporte al estudio de los geométridos de Chile.

\section{DISCUSIÓN}

Iridopsis hausmanni e I. parrai corresponden a las dos primeras especies de este género descritas de Chile.

La asociación entre estados inmaduros de Iridopsis y especies hospederas de Fabaceae, constatada en el presente estudio para I. parrai, ha sido previamente indicada para $I$. herse (Schaus), cuyos estados inmaduros han sido detalladamente descritos asociados a soya en Honduras (Passoa 1983).

La ausencia de una hilera de escamas piliformes sobre el tercer esternito del abdomen del macho, verificada en las dos especies aquí descritas, es una característica compartida únicamente por una especie Neotropical (Pitkin 2002), mientras que nueve especies Neárticas previamente incluidas en el Grupo I de Anacamptodes McDunnough por Rindge (1966) también carecen de escamas piliformes sobre el tercer esternito abdominal del macho.

Por otro lado, la morfología genital del macho de $I$. hausmanni e I. parrai podría estar indicando una estrecha relación evolutiva entre ambas especies, conformando un grupo monofilético dentro de Iridopsis. Sin embargo, esta hipótesis deberá probarse con posteriores estudios filogenéticos.

Agradecimientos. Al Dr. Axel Hausmann, Zoologische Staatssammlung Muenchen, Alemania; y al Dr. Luis E. Parra, Universidad de Concepción, Chile, por proporcionar importantes comentarios sobre Iridopsis durante la preparación del manuscrito. Los comentarios y sugerencias de dos revisores anónimos sirvieron para mejorar el manuscrito.

\section{REFERENCIAS}

McGuffin, W. C. 1987. Guide to the Geometridae of Canada (Lepidoptera) II. Subfamily Ennominae. 4. Memoirs of the Entomological Society of Canada 138, $182 \mathrm{pp}$.

Passoa, S. 1983. Immature stages of Anacamptodes herse (Schaus) (Geometridae) on soybean in Honduras. Journal of the Lepidopterists' Society 37: 217-223.

Pitkin L. M. 2002. Neotropical Ennominae moths: a review of the genera (Lepidoptera: Geometridae). Zoological Journal of the Linnean Society 135: 121-401.

Rindge, F. H. 1966. A revision of the moth genus Anacamptodes (Lepidoptera, Geometridae). Bulletin of the American Museum of Natural History 132: 175-244. 